OPEN ACCESS

Edited by: Jerker Rönnberg, Linköping University, Sweden

Reviewed by: Amy Poremba, University of lowa, USA Klaus Mathiak RWTH Aachen University, Germany

*Correspondence: Takuya Kishida kishida.takuya0119@gmail.com

Specialty section: This article was submitted to Auditory Cognitive Neuroscience, a section of the journal Frontiers in Psychology

Received: 15 December 2015 Accepted: 29 March 2016 Published: 26 April 2016

Citation:

Kishida T, Nakajima Y, Ueda K and Remijn GB (2016) Three Factors Are Critical in Order to Synthesize Intelligible Noise-Vocoded Japanese Speech. Front. Psychol. 7:517. doi: 10.3389/fpsyg.2016.00517

\section{Three Factors Are Critical in Order to Synthesize Intelligible Noise-Vocoded Japanese Speech}

\author{
Takuya Kishida $^{1 *}$, Yoshitaka Nakajima ${ }^{2}$, Kazuo Ueda $^{2}$ and Gerard B. Remijn ${ }^{2}$ \\ ${ }^{1}$ Human Science, Graduate School of Design, Kyushu University, Fukuoka, Japan, ${ }^{2}$ Department of Human \\ Science/Research Center for Applied Perceptual Science, Kyushu University, Fukuoka, Japan
}

Factor analysis (principal component analysis followed by varimax rotation) had shown that 3 common factors appear across 20 critical-band power fluctuations derived from spoken sentences of eight different languages [Ueda et al. (2010). Fechner Day 2010, Padua]. The present study investigated the contributions of such power-fluctuation factors to speech intelligibility. The method of factor analysis was modified to obtain factors suitable for resynthesizing speech sounds as 20-critical-band noise-vocoded speech. The resynthesized speech sounds were used for an intelligibility test. The modification of factor analysis ensured that the resynthesized speech sounds were not accompanied by a steady background noise caused by the data reduction procedure. Spoken sentences of British English, Japanese, and Mandarin Chinese were subjected to this modified analysis. Confirming the earlier analysis, indeed 3-4 factors were common to these languages. The number of power-fluctuation factors needed to make noise-vocoded speech intelligible was then examined. Critical-band power fluctuations of the Japanese spoken sentences were resynthesized from the obtained factors, resulting in noise-vocoded-speech stimuli, and the intelligibility of these speech stimuli was tested by 12 native Japanese speakers. Japanese mora (syllable-like phonological unit) identification performances were measured when the number of factors was 1-9. Statistically significant improvement in intelligibility was observed when the number of factors was increased stepwise up to 6 . The 12 listeners identified $92.1 \%$ of the morae correctly on average in the 6 -factor condition. The intelligibility improved sharply when the number of factors changed from 2 to 3 . In this step, the cumulative contribution ratio of factors improved only by $10.6 \%$, from 37.3 to $47.9 \%$, but the average mora identification leaped from 6.9 to $69.2 \%$. The results indicated that, if the number of factors is 3 or more, elementary linguistic information is preserved in such noise-vocoded speech.

Keywords: speech perception, noise-vocoded speech, factor analysis, principal component analysis, critical band

\section{INTRODUCTION}

It is important to understand what acoustic characteristics of speech sounds are essential for speech intelligibility in order to elucidate the cognitive mechanisms of speech communication. The acoustic characteristics of speech that contribute to speech perception have been investigated with many different approaches. One of the most fruitful methods is to control acoustic characteristics of 
speech by signal processing and then to test the intelligibility of the synthesized signals (for reviews see Diehl et al., 2004; Samuel, 2011). The temporal change of spectra is the representative acoustic characteristic in this context, and is processed by a frequency analyzer of the auditory system (Plomp, 1964; Plomp and Mimpen, 1968; Plack, 2013).

Perceptual experiments in which spectral information was systematically degraded revealed that perceptual cues embedded in speech spectra are highly redundant (Remez et al., 1981; Baer and Moore, 1993; Shannon et al., 1995; Warren et al., 1995). These studies often proceeded from the concept of auditory filters (Patterson, 1974; Moore, 2012) or critical bands (Fletcher, 1940), indicating parallel channels to process frequency components. Although the widths of the critical bands were determined from behavioral data, each of them corresponds to a distance of about $1.3 \mathrm{~mm}$ along the basilar membrane (Fastl and Zwicker, 2006). There are about 20 critical bands in the commonly used frequency range of speech sounds, which means that we can use the power fluctuations in these frequency bands to perceive speech. In most situations, however, we can perceive speech sounds represented by a relatively small number of power fluctuations because of the redundancy of perceptual cues in speech sounds. Shannon et al. (1995) found that four bands of amplitude-modulated noise were sufficient for nearly perfect scores (>95\%) of word intelligibility. Many studies (e.g., Dorman et al., 1997; Loizou et al., 1999; Souza and Rosen, 2009; Ellermeier et al., 2015) have measured the intelligibility of noise-vocoded speech, and indicated results consistent with Shannon et al. (1995). These studies suggest that the 20 outputs of critical-band filters, for example, can be reduced to a smaller number of channels without sacrificing the speech intelligibility too much.

In the present study, the power fluctuations of speech signals in 20 critical-band filters were analyzed and resynthesized with a new method of factor analysis. This analysis method is a modification of principal component analysis followed by varimax rotation, and was developed to reduce the number of dimensions of observed variables while retaining the information conveyed by these variables as far as possible (Jolliffe, 2002).

One of the earliest studies that applied principal component analysis to speech sounds was conducted by Plomp et al. (1967). They found that 14 Dutch steady vowels were distinguishable on the first and second principal component plane; these first two principal components had a close relation with the first and the second formant of the vowels (Pols et al., 1973). Zahorian and Rothenberg (1981) performed principal component analyses of speech, and they suggested that 3-5 principal components might convey enough perceptual cues to make speech signals intelligible. In a more systematic study of Ueda et al. (2010), principal component analysis was followed by varimax rotation. They discovered that 3 common factors appeared in 20 critical-band power fluctuations derived from spoken sentences of eight different languages (American English, British English, Cantonese Chinese, French, German, Japanese, Mandarin Chinese, and Spanish). The same analysis was performed over speech samples from 15-, 20-, and 24month-old infants, and the 3 common factors observed in adult voices were gradually formed along with language acquisition (Yamashita et al., 2013).

Thus, 3 factors seem to reflect an acoustic language universal, and these factors may play important roles in speech perception. This speculation, however, was brought about only from observations of acoustic characteristics of speech sounds, and it was not yet clear whether the extracted factors convey any perceptual cues. In the present study, we therefore examined how many factors were needed to make speech signals sufficiently intelligible. If the first 3 factors would indeed make up a basic framework of speech perception, then speech sounds resynthesized from these factors should be intelligible enough. We thus performed a perceptual experiment employing resynthesized speech stimuli.

\section{SPEECH ANALYSIS}

The purpose of this analysis was to obtain power-fluctuation factors suitable for resynthesizing speech sounds.

\section{Materials}

Two-hundred speech sentences each spoken by five male native speakers of British English, 200 sentences each spoken by five male native speakers of Japanese, and 78 sentences $^{1}$ each spoken by five male native speakers of Mandarin Chinese were used in the present analysis. These materials were selected from a commercial speech database (NTT-AT., 2002), recorded digitally (16-bit linear quantization and sampling frequency of $16000 \mathrm{~Hz}$ ). The mean fundamental frequencies of the spoken sentences were $126 \mathrm{~Hz}(S D=30 \mathrm{~Hz})$ in British English, $136 \mathrm{~Hz}(S D=31 \mathrm{~Hz})$ in Japanese, and $164 \mathrm{~Hz}(S D=38 \mathrm{~Hz})$ in Mandarin Chinese. The three languages were chosen from the languages analyzed in the previous study of Ueda et al. (2010) as representatives of different families of languages. These three languages have different linguistic rhythms; English is a stress-timed language, Japanese is a mora-timed language, and Mandarin Chinese is a syllable-timed language (Ramus et al., 1999).

\section{Procedure}

Speech sentences were resampled every $1 \mathrm{~ms}$ with a 30 ms-long Hamming window. From the extracted short time segments, power spectra were obtained through a Fast Fourier Transformation (FFT). Following this, these power spectra were smoothed with a 5-ms shortpass lifter by cepstral analysis (for a review on cepstral analysis see Rabiner and Schafer, 1978) to remove unnecessary details of the spectra. A 5-ms shortpass lifter removed fine structure of the power spectra narrower than $200 \mathrm{~Hz}$ that reflected vocal folds vibrations. Smoothed power spectra were then divided into 20 critical bands, and averaged power was calculated for each band. Thus, 20 temporal power fluctuations were obtained. The 20 critical bandwidths were taken from Zwicker and Terhardt (1980). The bandwidths originally ranged from 0 to $6400 \mathrm{~Hz}$, but since the range below

\footnotetext{
${ }^{1}$ The number of Mandarin Chinese sentences was smaller than that of British English sentences or Japanese sentences because speech files with technical problems in the recordings were excluded from the analysis.
} 
$50 \mathrm{~Hz}$ is unrelated to speech, the 1st bandwidth was narrowed from $[0-100 \mathrm{~Hz}]$ to $[50-100 \mathrm{~Hz}]$ (Table 1).

The 20 power fluctuations were subjected to a new type of principal component analysis followed by varimax rotation. Origin-shifted principal component analysis as used in this study proceeds from the idea that calculated eigenvectors should originate not from the gravity center of the data but from the zero point ${ }^{2}$, i.e., acoustically silent point. If the silent point is not contained in the subspace of the principal components, resynthesized sounds should generate noise even at the point corresponding to the silent point. In other words, the silent point is mapped onto a point indicating a certain acoustic power. As a result, the listener perceives a steady background noise in the resynthesized speech sounds (probably, this kind of steady background noise should have appeared in Zahorian and Rothenberg's (1981) resynthesized speech). An example of a steady background noise in a resynthesized speech sound is shown in Figure 1.

The eigenvectors derived with origin-shifted principal component analysis were rotated by varimax rotation (Kaiser, 1958), resulting in power-fluctuation factors. The purpose of varimax rotation was to make the relation between the factors and the critical bands easier to interpret because the orthogonality of the factors was maintained. The total number of factors produced in the above procedure was varied from 1 to 9 (for example, when 3 power-fluctuation factors were obtained, the eigenvectors of the first 3 principal components were rotated).

\section{Results and Discussion}

Figure 2 shows the cumulative contributions of the first 19 principal components. Over $70 \%$ variance of the power fluctuations was accounted for by the first 9 principal components in all three languages $(75,76$, and $71 \%$ for British English, Japanese, and Mandarin Chinese, respectively). A plausible explanation for the lower cumulative contributions for Mandarin Chinese is that the mean fundamental frequency of Mandarin Chinese speech was higher than that of the other languages, and that the cepstral liftering could not smooth the power spectra sufficiently.

Figure 3 shows factor loadings obtained with the three languages. The patterns of the power-fluctuation factors were similar among the three languages when the number of extracted factors was up to 4 (Figures $\mathbf{3 A - C}, \mathbf{E}-\mathbf{G}, \mathbf{I}-\mathbf{K}$ ). The cumulative contributions of the 4 power-fluctuation factors were 53, 55, and $48 \%$ for British English, Japanese, and Mandarin Chinese, respectively. When the number of factors was 5 or larger, it was difficult to find similar patterns of factors among these languages (Figures 3D,H,L). This means that about $50 \%$ of variance in the 20 power fluctuations could be mapped onto a common subspace of 4 fluctuation factors for the three languages.

In the 3- and 4-factor analyses, the factors seemed to divide speech sounds into four frequency bands (about $50-550 \mathrm{~Hz}$,

${ }^{2}$ Only when the gravity center of the data is identical with the zero point, the eigenvectors in the usual sense originate from the zero point. To realize this special case, principal component analysis was performed on the power fluctuations connected with their sign-reversed counterparts.
TABLE 1 | Critical bands for analysis.

\begin{tabular}{|c|c|c|}
\hline Band no. & Center frequency $(\mathrm{Hz})$ & Passband (Hz) \\
\hline 1 & 75 & $50-100$ \\
\hline 2 & 150 & $100-200$ \\
\hline 3 & 250 & $200-300$ \\
\hline 4 & 350 & $300-400$ \\
\hline 5 & 450 & $400-510$ \\
\hline 6 & 570 & $510-630$ \\
\hline 7 & 700 & $630-770$ \\
\hline 8 & 840 & $770-920$ \\
\hline 9 & 1000 & $920-1080$ \\
\hline 10 & 1170 & $1080-1270$ \\
\hline 11 & 1370 & $1270-1480$ \\
\hline 12 & 1600 & $1480-1720$ \\
\hline 13 & 1850 & 1720-2000 \\
\hline 14 & 2150 & 2000-2320 \\
\hline 15 & 2500 & 2320-2700 \\
\hline 16 & 2900 & 2700-3150 \\
\hline 17 & 3400 & $3150-3700$ \\
\hline 18 & 4000 & $3700-4400$ \\
\hline 19 & 4800 & $4400-5300$ \\
\hline 20 & 5800 & $5300-6400$ \\
\hline
\end{tabular}

The center frequency (not necessarily the exact mathematical center) and cutoff frequencies were adopted from Zwicker and Terhardt (1980), except for the lowest band.

$550-1700 \mathrm{~Hz}, 1700-3500 \mathrm{~Hz}$, and over $3500 \mathrm{~Hz}$ ). One of the factors obtained in the 3-factor analyses had high loadings at two frequency bands, the 1 st and the $3 \mathrm{rd}$ band. These bimodal factors were first reported by Ueda and Nakajima (2008) and Ueda et al. (2010), in which they predicted that the bimodal factor would be separated into 2 factors if they could elaborate the analysis method. The predicted factors indeed appeared in the 4 -factor analysis.

\section{SPEECH INTELLIGIBILITY EXPERIMENT}

The purpose of this experiment was to determine the number of power-fluctuation factors needed to make speech sufficiently intelligible. We chose Japanese speech sentences as the basis for sound stimuli. Japanese is convenient for scoring answers reported by participants because Japanese words can be broken up into morae, which are syllable-like phonological units. Each lexical mora is uniquely represented by a single Japanese "hiragana" letter used in writing.

\section{Participants}

Six men and six women, ranging in age from 19 to 24 years old (mean age $=21.5$ years, $S D=1.6$ years), participated as volunteers. They were all native speakers of Japanese with puretone thresholds lower than $25 \mathrm{~dB} \mathrm{HL}$ at audiometric frequencies of $125-8000 \mathrm{~Hz}$ for both ears. They were naive as to the purpose of the experiment. The procedure of the experiment was approved by the Ethics Committee of the Faculty of Design, Kyushu University. All participants provided written informed consent as to their participation. 

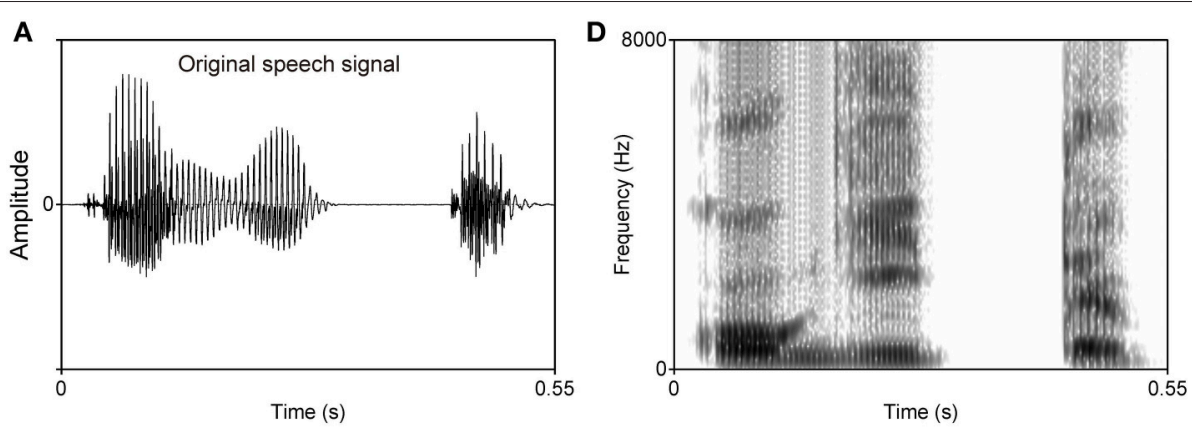

B

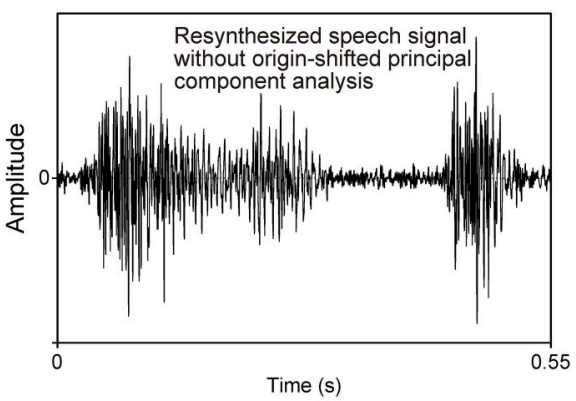

C

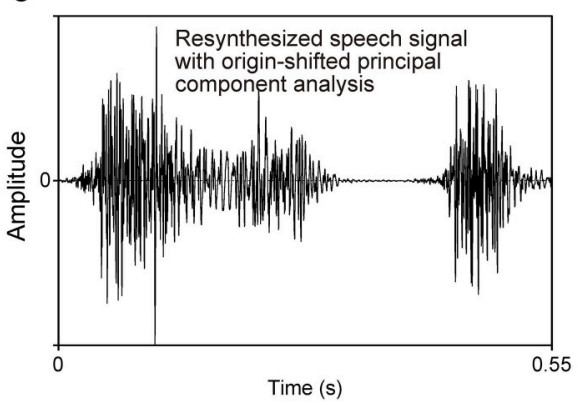

E

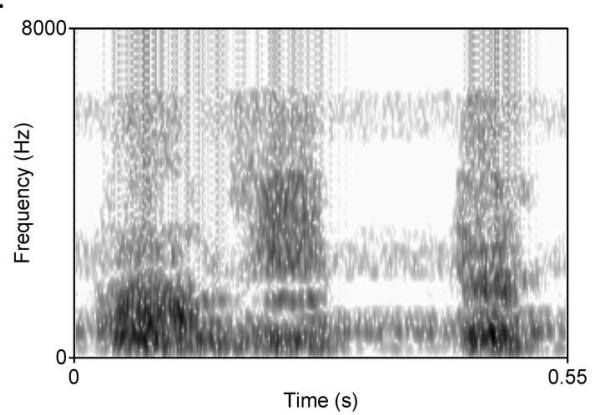

$\mathbf{F}$

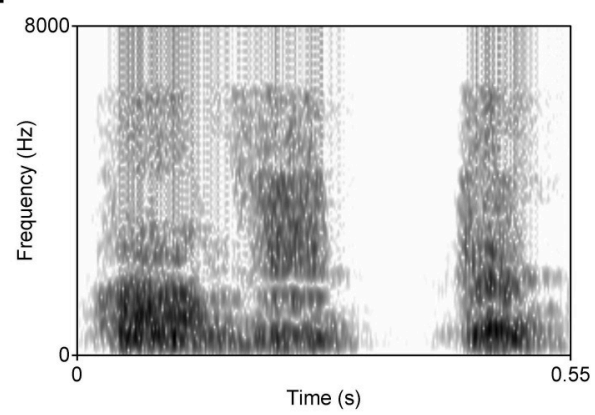

FIGURE 1 | Waveforms (left column) and spectrograms (right column) of an original speech signal (A,D), the resynthesized speech signal without origin-shifted principal component analysis $(\mathrm{B}, \mathrm{E})$, and the resynthesized speech signal with origin-shifted principal component analysis as proposed here (C,F). Steady background noise is observed in the speech signal resynthesized from the factors obtained with normal principal component analysis followed by varimax rotation $\mathbf{( B , E ) , ~ b u t ~ n o ~ s u c h ~ n o i s e ~ a p p e a r s ~ i n ~ t h e ~ s p e e c h ~ s i g n a l ~ r e s y n t h e s i z e d ~ f r o m ~ t h e ~ f a c t o r s ~ o b t a i n e d ~ w i t h ~ o r i g i n - s h i f t e d ~ p r i n c i p a l ~ c o m p o n e n t ~ a n a l y s i s ~}$ followed by varimax rotation $\mathbf{( C , F ) . ~}$

\section{Equipment}

The experiment was conducted in a sound-proof room, where the background noise level was below $25 \mathrm{dBA}$. The sound stimuli were generated digitally (16-bit linear quantization and sampling frequency of $16000 \mathrm{~Hz}$ ), with a computer (Frontier KZFM71/N) equipped with an audio card (E-MU 0404). The sounds were presented binaurally (diotically) to the participant via a digitalto-analog converter (ONKYO, SE-U55GX), an active low-pass filter (NF DV-04 DV8FL, cutoff at $7000 \mathrm{~Hz}$ ), a digital graphic equalizer (Roland, RDQ-2031), an amplifier (STAX, SRM-323S), and headphones (STAX, SR-307). The active low-pass filter was for avoiding aliasing, and the digital graphic equalizer was to equalize frequency responses of the headphones.

\section{Stimuli}

Original speech signals for sound stimuli were digitally recorded Japanese sentences (16-bit linear quantization and sampling frequency of $16000 \mathrm{~Hz}$ ), selected from a commercial speech database (NTT-AT., 2002). Fifty-seven sentences, each containing 17 to 19 morae (mean $=18$ morae), spoken by a male speaker were used; nine sentences were used for training trials, three sentences for warm-up trials, and the remaining 45 sentences for measurement trials. These sentences were part of the 200 sentences used to determine the power-fluctuation factors in the analysis.

The original speech signals were resynthesized from factors as 20-band noise-vocoded speech. The number of factors was 1-9 resulting in nine conditions. The 45 sentences used for measurement trials were divided into nine lists, each containing five sentences of 17 to 19 morae (mean $=18$ morae). Each list was assigned to a different factor-number condition, and the assignment of the sentence lists to the factor-number conditions was different among participants (Table 2). The nine sentences for training trials were also assigned to different 
TABLE 2 | The assignment of sentence lists to the factor number conditions for each participant.

\begin{tabular}{|c|c|c|c|c|c|c|c|c|c|}
\hline \multicolumn{10}{|c|}{ Sentence list } \\
\hline & A & B & C & D & $\mathbf{E}$ & $\mathbf{F}$ & G & $\mathbf{H}$ & I \\
\hline Participant I & 1 & 2 & 3 & 4 & 5 & 6 & 7 & 8 & 9 \\
\hline Participant II & 9 & 1 & 2 & 3 & 4 & 5 & 6 & 7 & 8 \\
\hline Participant III & 8 & 9 & 1 & 2 & 3 & 4 & 5 & 6 & 7 \\
\hline Participant IV & 7 & 8 & 9 & 1 & 2 & 3 & 4 & 5 & 6 \\
\hline Participant V & 6 & 7 & 8 & 9 & 1 & 2 & 3 & 4 & 5 \\
\hline Participant VI & 5 & 6 & 7 & 8 & 9 & 1 & 2 & 3 & 4 \\
\hline Participant VII & 4 & 5 & 6 & 7 & 8 & 9 & 1 & 2 & 3 \\
\hline Participant VIII & 3 & 4 & 5 & 6 & 7 & 8 & 9 & 1 & 2 \\
\hline Participant IX & 2 & 3 & 4 & 5 & 6 & 7 & 8 & 9 & 1 \\
\hline Participant X & 9 & 8 & 7 & 6 & 5 & 4 & 3 & 2 & 1 \\
\hline Participant XI & 8 & 7 & 6 & 5 & 4 & 3 & 2 & 1 & 9 \\
\hline Participant XII & 7 & 6 & 5 & 4 & 3 & 2 & 1 & 9 & 8 \\
\hline
\end{tabular}

The number from 1 to 9 indicates the factor number condition. Each sentence list consisted of five sentences containing 17-19 morae each.

factor-number conditions, but the assignment of sentences to the conditions was the same among participants. The three sentences used for warm-up trials were of 7- to 9-factor conditions.

In order to synthesize noise-vocoded speech, the reproduced 20 power fluctuations of the original speech signal were used. With the same procedure as described in the Speech Analysis section, 20 power fluctuations were extracted from the original speech signal. To obtain time series of factor scores, the score of the $t$ th time frame of the $k$ th factor, $X_{k, t}$ was calculated by the following equation:

$$
X_{k, t}=\sum_{n=1}^{20} A_{k, n} Y_{n, t},
$$

where $A_{k, n}$ is the $n$th component of the normalized vector indicating the $k$ th factor of the $K(=1 \ldots 9)$ power-fluctuation factor(s) determined in the analysis of Japanese speech in the Speech Analysis section, and $Y_{n, t}$ is the $t$ th time frame of the power fluctuation in the $n$th critical band. $A_{k, n}$ was different between the factor-number conditions as is plotted in Figure 4. Next, 20 power fluctuations were reproduced by

$$
\hat{Y}_{n, t}=\sum_{k=1}^{K} A_{k, n} X_{k, t},
$$

where, $\hat{Y}_{n, t}$ is the $t$ th time frame of the reproduced power fluctuation in the $n$th critical band. Geometrically, the transformations can be regarded as the projections of 20 power fluctuations in a 20-dimensional Euclidean space onto the $K$-dimensional subspace formed by the normalized vectors indicating the obtained factors.

White noise was generated, and was passed through banks of digital filters with the same cutoff frequencies as specified in Table 1. Twenty power fluctuations were then computed by squaring and smoothing each bandpass-filter output. The ratio

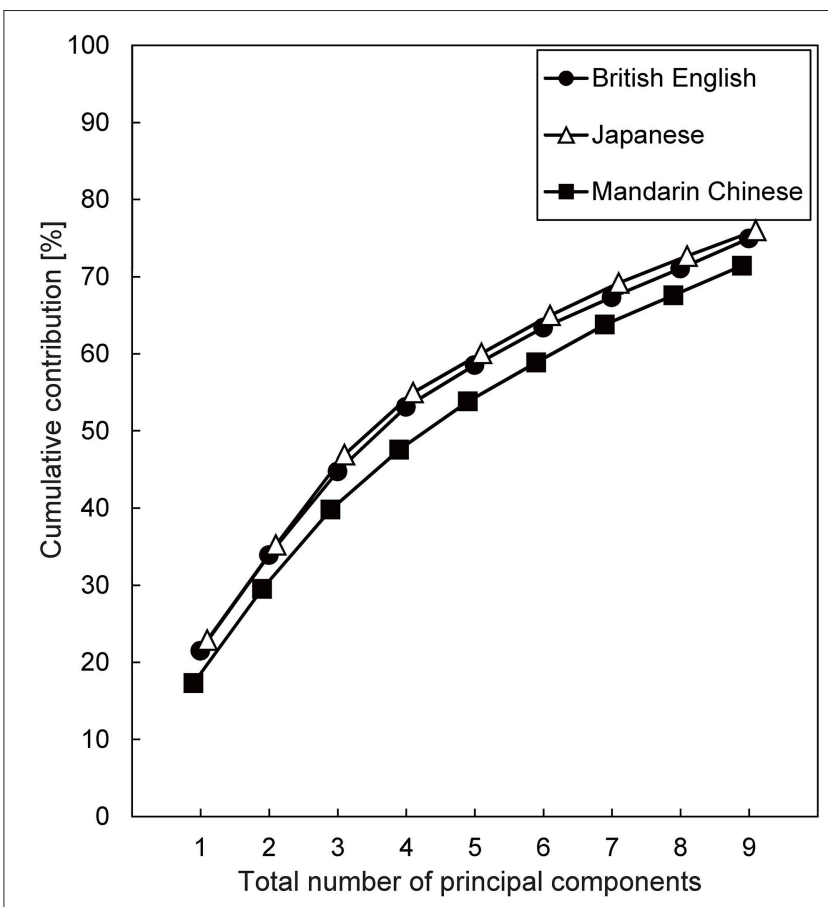

FIGURE 2 | The cumulative contribution as a function of the number of principal components for power fluctuations in the $\mathbf{2 0}$ critical-band filters. Spoken sentences of three languages were investigated.

between the reproduced power of the original speech signal as in equation (2) and the power of the generated noise was calculated in each critical band at each sample point ${ }^{3}$. The 20 bandpassfiltered noises were thus modulated with that ratio to realize the 20 reproduced power fluctuations of speech sound. Finally, the modulated bandpass-filtered noises were added up to yield noise-vocoded speech.

\section{Procedure}

The intelligibility experiment started with one training block of nine trials, followed by three main blocks which each consisted of one warm-up trial and 15 measurement trials. The participant, who sat on a chair in front of the computer screen wearing headphones, was asked to click a "play" button on the screen for each trial. A sound stimulus was presented $0.5 \mathrm{~s}$ after the button was clicked. The presentation was repeated three times with 1.5$s$ intervals. After listening to the sound stimulus, the participant typed the morae (syllable-like phonological units) which he/she heard using hiraganas (Japanese moraic phonograms). The participant was instructed to avoid guessing parts of sentences which were not heard clearly. All stimuli in main blocks were presented in random order.

\section{Results and Discussion}

Figure 5 shows the percentage of mora identification as a function of the number of factors used to reconstruct the 20 power fluctuations of the Japanese speech stimuli. Mora

${ }^{3}$ The sample size of the 20 reproduced power fluctuations was increased by repeating values to equate with the sample size of the power fluctuations of the bandpass-filtered noises. 


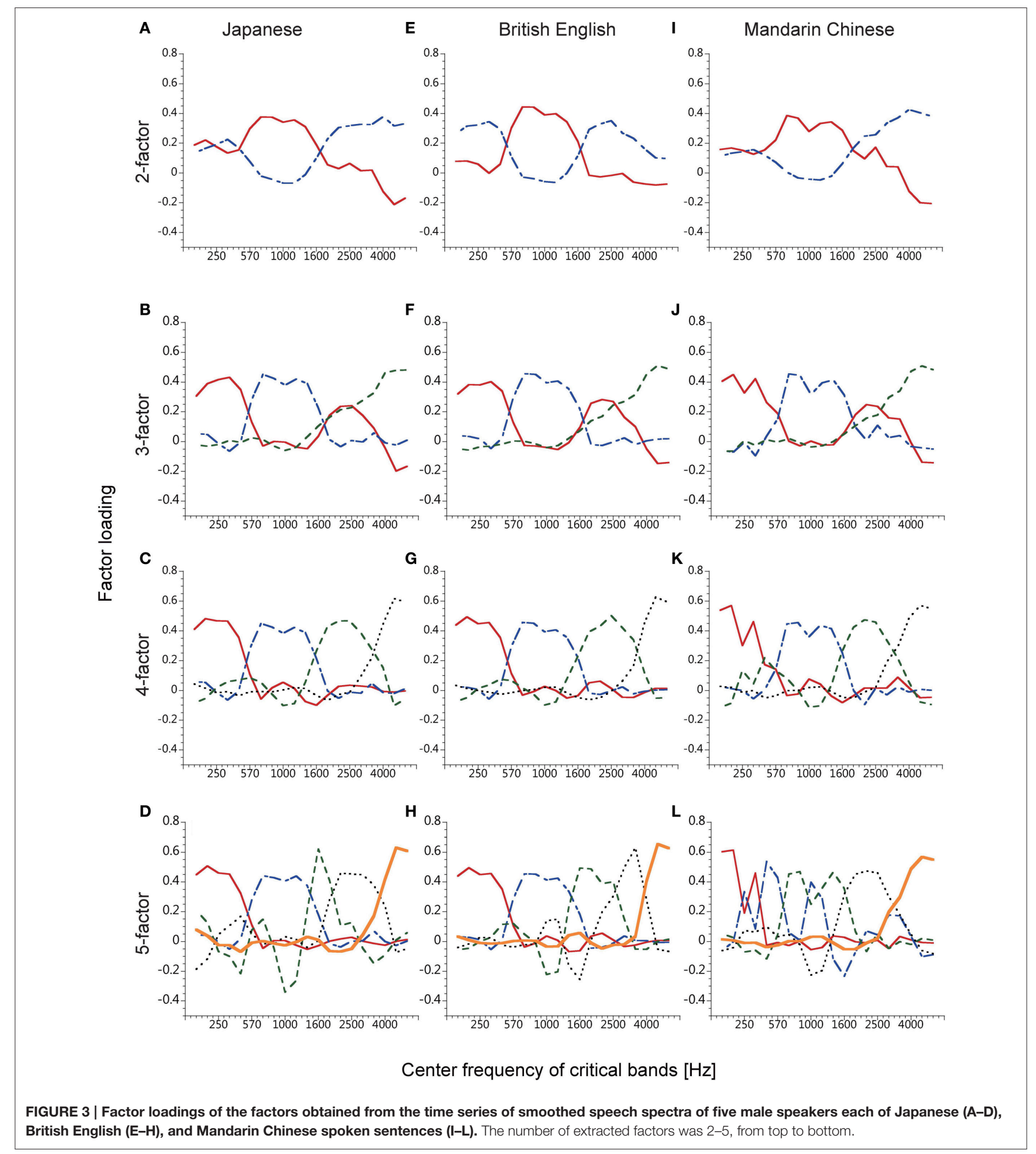

identification increased with the number of factors, and approached a plateau around the 4-factor condition, where the participants' performance was $83.7 \%(S D=5.8 \%)$. Mora identification was subjected to arcsine transformation, and a oneway Analysis of Variance (ANOVA) with repeated measures was performed. The results showed that the main effect of number of factors was significant $\left[F_{(8,88)}=315.44, p<0.0001\right]$. Posthoc tests according to Scheffe showed no statistically significant differences in mora identification when the number of factors was increased beyond $6\left[F_{(8,99)}=3.83, p=0.869\right.$, n.s. $]$. There was a significant difference $\left[F_{(8,99)}=317.36, p<0.001\right]$ in the mora identification between the 2 - and the 3 - [or more] 


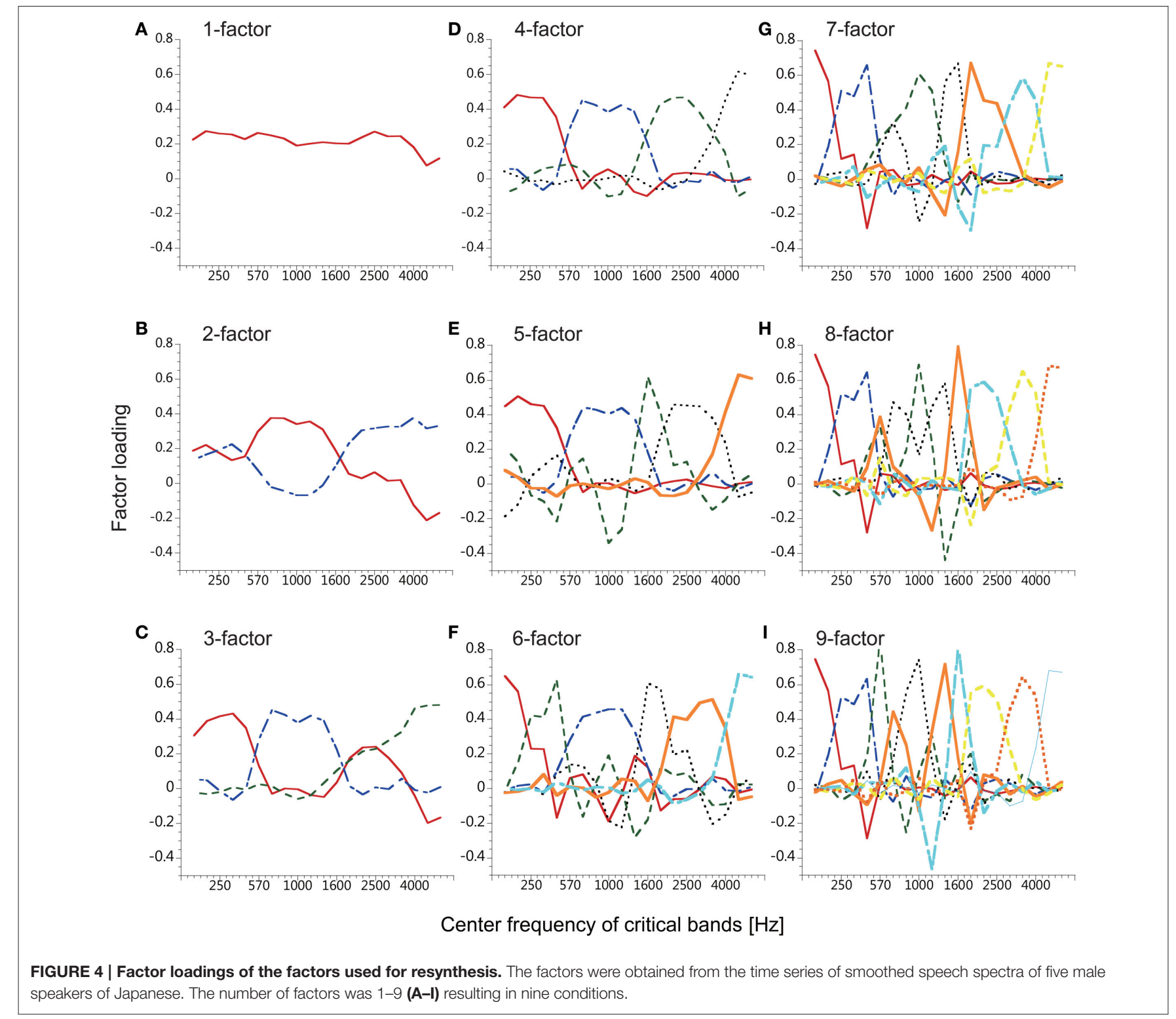

factor conditions, and between the 3- and the 4- [or more] factor conditions $\left[F_{(8,99)}=16.68, p<0.05\right]$. There was no significant difference between the mora identifications obtained with the 4and the 5- factor condition $\left[F_{(8,99)}=1.37, p=0.994\right.$, n.s.], between the 4 - and the 6 -factor condition $\left[F_{(8,99)}=11.09\right.$, $p=0.212$, n.s.], or between the 5- and the 8- factor condition $\left[F_{(8,99)}=10.15, p=0.269\right.$, n.s. $]$. A remarkable improvement of mora identification appeared when the number of factors was changed from 2 to 3 ; the average mora identification leaped from $7 \%$ to about $70 \%$ [exactly from $6.9 \%(S D=6.7 \%)$ to $69.2 \%(S D=$ $11.7 \%)]$. The first 3 factors turned out to be critical for speech intelligibility.

\section{GENERAL DISCUSSION}

We applied factor analysis to spoken sentences in three different languages, and performed an intelligibility test of
Japanese sentences to investigate how power-fluctuation factors contributed to speech perception. The method of the factor analysis used in a previous study (Ueda et al., 2010) was modified in order to make it possible to resynthesize power fluctuations of speech across 20 critical bands from the obtained factors. The power-fluctuation factors extracted with this modified analysis method had very similar profiles to the ones in the previous studies (Ueda et al., 2010; Ellermeier et al., 2015). Twenty critical bands were divided into four frequency regions by the factors when the number of extracted factors was 3 or 4 . These factors appeared commonly across three languages, i.e., British English, Japanese, and Mandarin Chinese. This was consistent with the results of Ueda et al. (2010). The drastic modification of the analysis method did not distort the essential features of the factors. The advantage of the present modification was that the vectors indicating the factors originated from the acoustically silent point. The silent point mapped onto the 


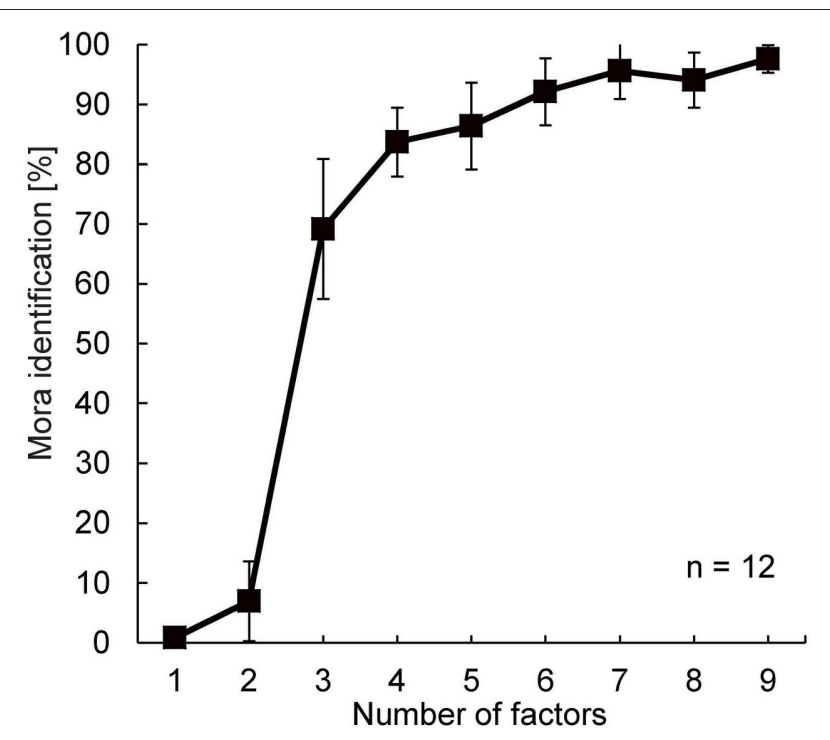

FIGURE 5 | Results of the speech intelligibility experiment. The $x$-axis indicates the number of factors employed for reconstructing the 20 power fluctuations of the original speech sounds. The $y$-axis indicates mora identification. Error bars show standard deviations.

extracted subspace generated no noise: Silent parts remained silent when resynthesized.

The set of the 3 power-fluctuation factors proved to play a vital role in making speech intelligible. Although the 3 factors explained only $47.9 \%$ of the power fluctuations in the original speech sentences, $69.2 \%(S D=11.7 \%)$ of the morae in the Japanese sentences were conveyed perceptually through these factors. Less than a half of the physical variance thus is very likely to be more informative than the rest. The finding that the 6-factor condition finally led to an asymptotic performance suggests that the information in the 3-4 factors forms the basis of perceptual cues, but that it is not yet sufficient to carry phonological details.

Let us compare the participants' performance in this study with that in four previous studies (Shannon et al., 1995; Dorman et al., 1997; Souza and Rosen, 2009; Ellermeier et al., 2015), which investigated the relationship between the number of vocoding channels and sentence recognition. Four-channel noise-vocoded

\section{REFERENCES}

Baer, T., and Moore, B. C. (1993). Effects of spectral smearing on the intelligibility of sentences in noise. J. Acoust. Soc. Am. 94, 1229-1241. doi: 10.1121/1.408176

Diehl, R. L., Lotto, A. J., and Holt, L. L. (2004). Speech perception. Annu. Rev. Psychol. 55, 149-179. doi: 10.1146/annurev.psych.55.090902.142028

Dorman, M. F., Loizou, P. C., and Rainey, D. (1997). Speech intelligibility as a function of the number of channels of stimulation for signal processors using sine-wave and noise-band outputs. J. Acoust. Soc. Am. 102, 2403-2411. doi: $10.1121 / 1.419603$

Ellermeier, W., Kattner, F., Ueda, K., Doumoto, K., and Nakajima, Y. (2015). Memory disruption by irrelevant noise-vocoded speech: effects of native language and the number of frequency bands. J. Acoust. Soc. Am. 138, 1561-1569. doi: 10.1121/1.4928954

Fastl, H., and Zwicker, E. (2006). Psychoacoustics: Facts and Models, 3rd Edn. Heidelberg: Springer. speech induced high intelligibility in these previous studies (95\% correct score in Shannon et al., 1995; Dorman et al., 1997; Ellermeier et al., 2015, and 70\% correct score in Souza and Rosen, 2009). These correct scores are not too far from those obtained in the 3- and the 4-factor condition in our experiment. Very probably, four-channel noise-vocoded speech of which bandwidths are determined by the 3 or 4 factors in the present paradigm will be intelligible as well (see Ellermeier et al., 2015). The reason why high recognition performances were obtained with four-channel noise-vocoded speech in the previous studies can be explained if we assume that the fundamental nature of speech sounds consists of 3 or 4 bands of power fluctuations, and originates from constraints of the size and structure of the human articulatory organs (Yamashita et al., 2013, Figure A3). This conjecture comes from the fact that 3-4 factors commonly appeared in three different languages, and speech perception seems to be based on the perceptual cues carried by the 3-4 power-fluctuation factors.

If the power-fluctuation factors obtained in this study play an essential role in human speech communication, some correspondence will be found between the factors and articulatory movements as well as brain activities related to speech communication. The present findings might contribute to the development of technology supporting speech communication on various occasions.

\section{AUTHOR CONTRIBUTIONS}

TK and YN designed the study. TK collected and analyzed the data, and the other authors supported him occasionally. All the authors interpreted the results together. TK wrote the first draft, and all the authors improved the paper together. YN gave the final approval of the version to be published.

\section{ACKNOWLEDGMENTS}

This study was supported by a JSPS KAKENHI Grant-in-Aid for Scientific Research (A) (25242002), and a JSPS KAKENHI Grantin-Aid for Exploratory Research (26540145). Asuka Ono gave us technical assistance.

Fletcher, H. (1940). Auditory patterns. Rev. Modern Phys. 12, 47-65. doi: 10.1103/RevModPhys.12.47

Jolliffe, I. (2002). Principal Component Analysis. New York, NY: Springer.

Kaiser, H. F. (1958). The varimax criterion for analytic rotation in factor analysis. Psychometrika 23, 187-200. doi: 10.1007/BF02289233

Loizou, P. C., Dorman, M., and Tu, Z. (1999). On the number of channels needed to understand speech. J. Acoust. Soc. Am. 106, 2097-2103. doi: $10.1121 / 1.427954$

Moore, B. C. J. (2012). An Introduction to the Psychology of Hearing, 6th Edn. London: Academic Press.

NTT-AT. (2002). Multilingual Speech Database 2002. Tokyo: NTT Advanced Technology Corporation. Available online at: http://www.ntt-at.com/product/ speech2002/ (Accessed December 1, 2015).

Patterson, R. D. (1974). Auditory filter shape. J. Acoust. Soc. Am. 55, 802-809. doi: $10.1121 / 1.1914603$

Plack, C. J. (2013). The Sense of Hearing. New York, NY: Psychology Press. 
Plomp, R. (1964). The ear as a frequency analyzer. J. Acoust. Soc. Am. 36, 1628-1636. doi: 10.1121/1.1919256

Plomp, R., and Mimpen, A. (1968). The ear as a frequency analyzer. II. J. Acoust. Soc. Am. 43, 764-767. doi: 10.1121/1.1910894

Plomp, R., Pols, L. C. W., and van de Geer, J. P. (1967). Dimensional analysis of vowel spectra. J. Acoust. Soc. Am. 41, 707-712. doi: 10.1121/1.1910398

Pols, L. C. W., Tromp, H. R. C., and Plomp, R. (1973). Frequency analysis of Dutch vowels from 50 male speakers. J. Acoust. Soc. Am. 53, 1093-1101. doi: 10.1121/1.1913429

Rabiner, L. R., and Schafer, R. W. (1978). Digital Processing of Speech Signals. Englewood Cliffs, NJ: Prentice Hall.

Ramus, F., Nespor, M., and Mehler, J. (1999). Correlates of linguistic rhythmin the speech signal. Cognition 73, 265-292. doi: 10.1016/S0010-0277(99)00058-X

Remez, R. E., Rubin, P. E., Pisoni, D. B., and Carrell, T. D. (1981). Speech perception without traditional speech cues. Science 212, 947-949. doi: $10.1126 /$ science.7233191

Samuel, A. G. (2011). Speech perception. Annu. Rev. Psychol. 62, 49-72. doi: 10.1146/annurev.psych.121208.131643

Shannon, R. V., Zeng, F.-G., Kamath, V., Wygonski, J., and Ekelid, M. (1995). Speech recognition with primarily temporal cues. Science 270, 303-304. doi: $10.1126 /$ science.270.5234.303

Souza, P., and Rosen, S. (2009). Effects of envelope bandwidth on the intelligibility of sine-and noise-vocoded speech. J. Acoust. Soc. Am. 126, 792-805. doi: $10.1121 / 1.3158835$

Ueda, K., and Nakajima, Y. (2008). A consistent clustering of power fluctuations in British English, French, German, and Japanese. Trans. Tech. Comm. Psychol. Physiol. Acoust. 38, 771-776.
Ueda, K., Nakajima, Y., and Satsukawa, Y. (2010). "Effects of frequencyband elimination on syllable identification of Japanese noise-vocoded speech: analysis of confusion matrices," in Fechner Day 2010, Proceedings of the 26th Annual Meeting of the International Society for Psychophysics (Padova), 39-44.

Warren, R. M., Riener, K. R., Bashford, J. A., and Brubaker, B. S. (1995). Spectral redundancy: intelligibility of sentences heard through narrow spectral slits. Percept. Psychophys. 57, 175-182. doi: 10.3758/BF03206503

Yamashita, Y., Nakajima, Y., Ueda, K., Shimada, Y., Hirsh, D., Seno, T., et al. (2013). Acoustic analyses of speech sounds and rhythms in Japanese-and English-learning infants. Front. Psychol. 4:57. doi: 10.3389/fpsyg.2013.00057

Zahorian, S. A., and Rothenberg, M. (1981). Principal-components analysis for low-redundancy encoding of speech spectra. J. Acoust. Soc. Am. 69, 832-845. doi: $10.1121 / 1.385539$

Zwicker, E., and Terhardt, E. (1980). Analytical expressions for critical-band rate and critical bandwidth as a function of frequency. J. Acoust. Soc. Am. 68, 1523-1525. doi: 10.1121/1.385079

Conflict of Interest Statement: The authors declare that the research was conducted in the absence of any commercial or financial relationships that could be construed as a potential conflict of interest.

Copyright (c) 2016 Kishida, Nakajima, Ueda and Remijn. This is an open-access article distributed under the terms of the Creative Commons Attribution License (CC $B Y)$. The use, distribution or reproduction in other forums is permitted, provided the original author(s) or licensor are credited and that the original publication in this journal is cited, in accordance with accepted academic practice. No use, distribution or reproduction is permitted which does not comply with these terms. 